The Foreground Bias: Initial Scene Representations across the Depth Plane

\author{
Suzette Fernandes and Monica S. Castelhano \\ Department of Psychology \\ Queen's University
}

Please address correspondence to:

Monica S. Castelhano

Department of Psychology

Queen's University

Kingston ON K7L 3N6

Canada

Phone: 613 533-3266

Email: monica.castelhano@queensu.ca 


\begin{abstract}
When you walk into a large room, you perceive visual information that is both close to you in depth and farther in the background. Here, we investigated how initial scene representations are affected by information across depth. We examined the role of background and foreground information on scene gist by using Chimera scenes (images with foreground and background from different scene categories). Across three experiments, we found a Foreground Bias in which foreground information initially had a strong influence on the interpretation of the scene. This bias persisted when the initial fixation position was on the scene background and when the task was changed to emphasize scene information. We conclude that the Foreground Bias arises from initial processing of scenes for understanding and suggests that scene information closer to the observer is initially prioritized. We discuss the implications for theories of scene and depth perception.
\end{abstract}

Keywords: rapid scene gist perception, scene categorization, depth, contextual bias 


\section{The Foreground Bias: Initial Scene Representations across the Depth Plane}

Imagine walking into a room for the first time. Rapidly, you understand the size and shape of the space, different objects, and other visual features. Researchers have known for decades that the overall meaning of a scene is quickly extracted (Monica S. Castelhano \& Henderson, 2008; Greene \& Oliva, 2009b; Potter, 1975, 1976). In a seminal study, Potter (1975) first demonstrated that humans were able to ascertain a scene's general meaning (scene gist) with as little as $113 \mathrm{~ms}$ of exposure, and since then other researchers have shown scene understanding with even briefer presentations (e.g., 20-40ms, (Monica S. Castelhano \& Henderson, 2008; Greene \& Oliva, 2009b; Thorpe, Fize, \& Marlot, 1996). Subsequent studies on scene gist have explored how different sources of information contribute to rapid scene understanding.

\section{Scene Gist Processing}

Studies examining rapid scene understanding have parsed visual information in various ways. For instance, Schyns and Oliva (1994) posited that the information is extracted from a coarse-to-fine gradient. They presented participants with 'hybrid' images that were composed of a combination of the low-pass and high-pass information from separate images. When briefly presented, scenes were categorized according to low-frequency information, but to high-frequency with additional time. Additionally, Oliva and Schyns (1997) demonstrated that both low- and high-frequency information was available early on. They concluded scene gist is initially based on low-pass information, but prioritization is flexible (Oliva, 2005).

Researchers have also contrasted global scene structure with local visual details (de Graef et al., 1990; Henderson \& Hollingworth, 1999; Pereira \& Castelhano, 2014, 2019). Global information is defined as the overall shape of the scene (Greene \& Oliva, 2009a, 2010) or scene background (Davenport \& Potter, 2004; Munneke, Brentari, \& Peelen, 2013). Local visual details are often defined as the smaller moveable objects in the scenes (Henderson \& Hollingworth, 1999; Koehler \& 
Eckstein, 2017; Pereira \& Castelhano, 2014) or larger content objects (Castelhano, Fernandes, \& Theriault, 2018; Davenport \& Potter, 2004; Evans, Horowitz, \& Wolfe, 2011; Munneke et al., 2013). Although both influences are thought to affect the interpretation of scene gist, the general consensus in the literature is that scene gist is largely driven by global scene structure information. However, despite years of research on how scenes are processed, it is not yet clear how information across depth is initially perceived.

\section{Information across Depth}

Studies of depth perception have traditionally examined observers' estimates of distance (Cutting \& Vishton, 1995; Nagata, 1993) and how information at different distances are processed: from peripersonal to vista space (Costantini, Ambrosini, Scorolli, \& Borghi, 2011; Cutting \& Vishton, 1995; Previc, 1998). However, recent studies on scene processing have shown qualitatively different processing of spaces closer to the observer (Bonner \& Epstein, 2017, 2018; Josephs \& Konkle, 2019). For example, Bonner and Epstein (2017) have found that activity in the occipital place area (OPA) was linked to perceiving potential paths for movement in immediate surroundings. In addition, Josephs and Konkle (2019) have found that the representations of spaces that are reachable are qualitatively different than close-up objects and larger scene spaces. Given the qualitative differences in processing across depth, it stands to reason that information closer in depth may have different utility than information farther away and thus, may affect the initial processing of scene gist differently. Notably, this is in contrast to studies that suggest a primary role of the overall global structure on scene gist perception. Based on studies of depth perception, the present study examined whether information presented at different depths (foreground and background) differently affected the initial interpretation of scene. 


\section{Foreground and Background}

In the current study, scene information across depth was manipulated such that information in the foreground and background contained content from different scene categories (i.e. Chimera scenes, see Figure 1; Castelhano et al., 2018). We defined the background as the large boundary defining elements such as walls, floors, ceilings, and main structures that define the space (Oliva \& Torralba, 2001; Oliva, 2005) and the foreground as the content objects that were closer to the observer (Henderson \& Hollingworth, 1999; Oliva, 2005). We operationalized the distinction by manipulating scene category information (for instance, either from kitchen vs. living room) roughly at $50 \%$ of the overall depth of the room (foreground in the front half; background in the back half; see Methods). With this distinction, we examined how the scene gist perception is influenced by the background and foreground when each have different interpretations. 


\section{Kitchen}

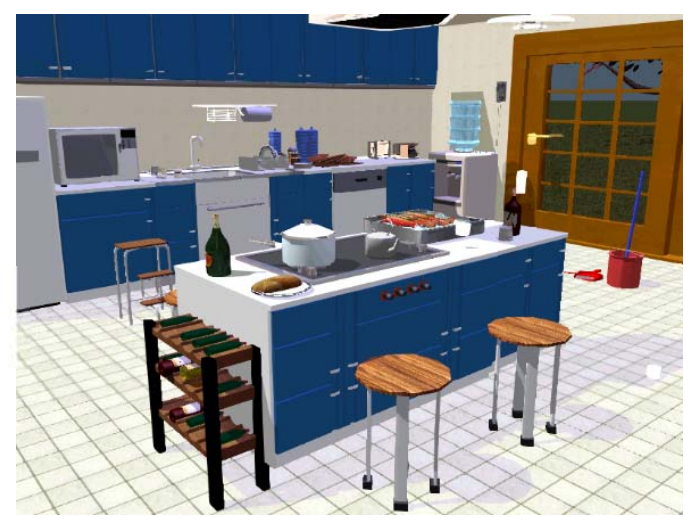

Normal

Chimera

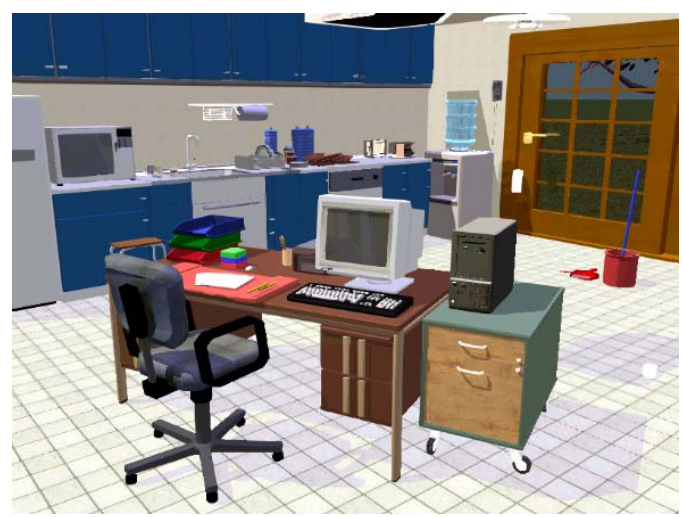

\section{Office}
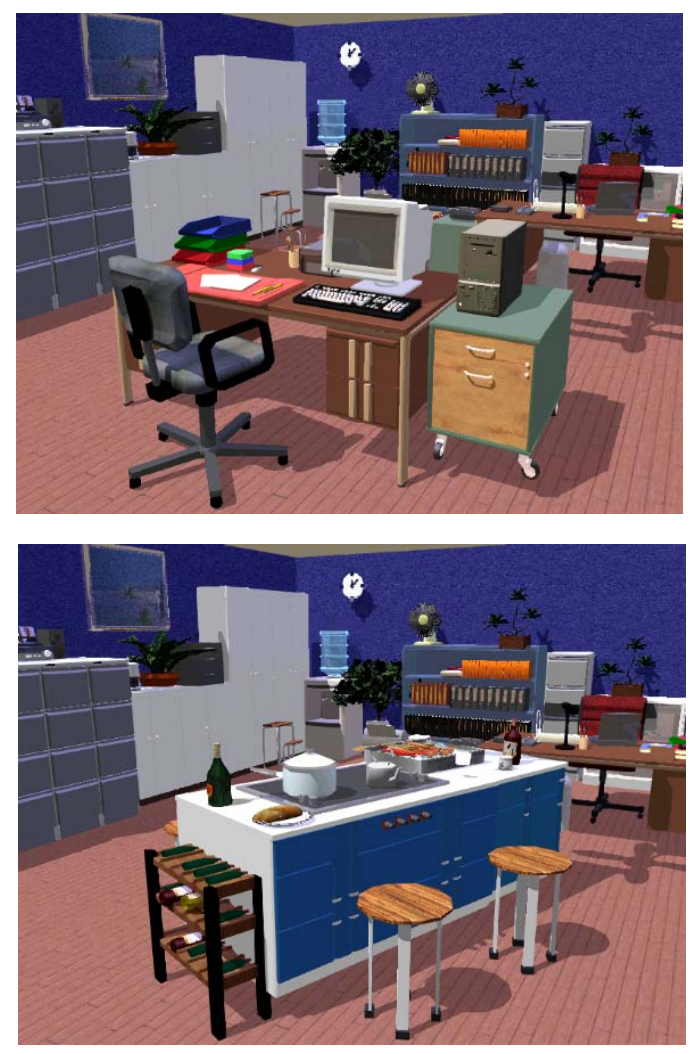

Figure 1. Two example stimuli used in both experiments. The top panel shows the Normal version of the scenes and the bottom panel shows the Chimera version of the scenes. Two images from separate categories were matched and the foreground objects were swapped to create the Chimera version for each background.

\section{Present study}

Across three experiments, participants were presented scenes with either foregrounds and backgrounds that were semantically matched (Normal scenes) or mismatched (Chimera scenes). To examine the interpretation of these scenes, we used a Contextual Bias paradigm (Monica S.

Castelhano \& Henderson, 2008), which allows for interpretation of the scene without relying on judgements of scene category names. With Contextual Bias paradigm, instead of verifying the scene category, participants indicated whether a target object was likely to be present. The Contextual Bias Paradigm reflects the participant's bias to confirm consistent target objects and to disconfirm inconsistent targets. 
In each of three experiments, we examined shorter $(50 \mathrm{~ms}$ or $100 \mathrm{~ms})$ and longer $(250 \mathrm{~ms}$ or 330ms) exposure durations in two sub-experiments. In Experiment 1, we examined performance when scenes were viewed from the image center. Experiment 2 investigated whether initial perception was influenced by fixation position. For each image, participants fixated either in the scene foreground or background. Experiment 3 examined the effect of task, and had participants respond to the scene category name. Across all experiments, we found that initially participants were strongly influenced by the scene foreground.

\section{Experiment 1}

\section{Methods}

Participants. Two groups of 36 participants took part in Experiments 1a and 1b. They were compensated with either course credit or an honorarium at the rate of $\$ 10 /$ hour. All participants had normal or corrected-to-normal vision. This study was cleared by Queen's University's General Research Ethics Board. Sample size was determined using the effect size for 50ms presentation (Castelhano \& Henderson, 2008), which had a medium to large effect size $(\mathrm{d}=$.6-2.7) depending on condition. Because we were interested in determining how manipulated scenes would affect interpretation, for our sample size computation (G*power, Faul, Erdfelder, Lang, \& Buchner, 2007) we used a conservative effect size of $.5, \alpha=.05$, and power at .8. The estimated sample size was 34 participants, but we used 36 to fit the counterbalancing (8 conditions), which yielded a power estimate of .83 .

Materials and Apparatus. Using HomeDesign 5.0 (Databecker, Germany), computer-generated coloured images consisting of both indoor and outdoor scene categories were created (see Figure 1 for example scenes). All scenes were created using the same template such they were built into the corner with two adjoining walls providing the main definition of the space and depth of the scene. Thus, indoor and outdoor scenes had a similar shape and depth. Each image's background and 
foreground area was then identified and partitioned ${ }^{1}$. The depth of the foreground information (from the camera position to the back wall occupied on average $52 \%(13 \% \mathrm{SD})$ of the distance. When rendered as 2D images, the pixel content for the foreground vs. background was roughly equated (51\% Foreground, 49\% Background).

To create the Chimera scenes, the foreground of one image was paired with a semantically different background of another image, and vice versa. The experimental stimuli consisted of 48 scenes, each with a Normal and Chimera version for a total of 96 images. The study was run using MATLAB R2014a and PsychToolBox3 (Brainard, 1997) on a 21"CRT monitor with a refresh rate of $100 \mathrm{~Hz}$ at a resolution of $800 \times 600$ pixels subtended $38.1^{\circ} \times 26.6^{\circ}$ with a refresh rate of $100 \mathrm{~Hz}$. Head position was restricted with a chinrest approximately $60 \mathrm{~cm}$ from the monitor.

Design. For each experiment, a 2 (presentation duration) x 2 (scene type: Normal, Chimera) x 3 (target condition: consistent-background, consistent-foreground, inconsistent) within-subjects design was used. The presentation duration was $50 \mathrm{~ms}$ and $100 \mathrm{~ms}$ for Experiment $1 \mathrm{a}$ and was $250 \mathrm{~ms}$ and 330ms for Experiment 1b. Participants saw 48 images total with an equal number of different scene types (24 Normal scenes and 24 Chimera scenes). The target word belonged to one of three conditions: (i) Consistent with the background scene category, (ii) Consistent with the foreground scene category, or (iii) Inconsistent with both the background and the foreground. Target objects were never present in the image.

Procedure. The procedure followed the Contextual Bias paradigm (Castelhano \& Henderson, 2008). Prior to beginning the experiment, they provided written informed consent. Across conditions participants were each tested individually. Participants were instructed to view briefly

\footnotetext{
${ }^{1}$ We had a separate group of participants $(\mathrm{N}=20)$ categorize either foreground or the background images rendered separately (background only or foreground only). We found that although Backgrounds (95\%) were categorized significantly more accurately $(t(18)=4.26, p<.01)$ than Foregrounds $(89 \%)$, both were categorized at a high level of accuracy.
} 
presented images and on the following query screen, make a response based on whether the target object named fit with the scene. Participants head was not retrained, but they sat approximately 60 $\mathrm{cm}$ from the monitor.

For each trial, participants were presented with a black fixation dot centred on a grey screen. An image then appeared on the screen for the duration corresponding with the duration condition. This was followed by a mask image for $50 \mathrm{~ms}$. The target word then appeared and participants indicated whether that target object was likely to be found in the image (Yes/No) via a response box. The target names were manipulated such that the object was consistent with either the foreground or background exclusively, or was inconsistent with both scene contexts. Each target object type was presented with equal frequency across trials. Importantly, as noted above, the queried target objects were never present in the scenes, so the task response did not reflect detection of the object, but rather the response bias of how likely the object was to appear in the scene. The logic is that if the foreground (or background) was perceived, then responding "yes" was more likely when the object was consistent and more unlikely when the target was inconsistent. Thus, responses were driven by the interpretation of the scene gist and thus gist perception is measured indirectly by examining the difference between the consistent and inconsistent responses. All images and conditions were counterbalanced across participants and participants saw each scene once. The experimental session lasted approximately 15-20 minutes.

\section{Results}

Analysis. To determine how participants attended to the foreground and the background of a scene, the mean proportion of times the participants responded "Yes" to the target object was calculated. This was used as the dependent variable for all reported analyses across all experiments. As each group of participants were collected at different times, each of the Experiment 1a and 1b 
analyses were conducted separately; however exploratory analysis across both experiments can be found in the Supplementary Material.

The main a priori analyses were aimed at investigating how the interpretation of the Chimera scenes changed over time. Based on this, we conducted pre-planned comparisons contrasting the responses of the foreground and background queries against the control condition (Inconsistent Target) as duration increased. We also planned contrasts between the responses to the foreground and background to examine whether they differed, at each exposure duration. This is interesting because in reality, both of these interpretations are legitimate. However, responses that favour one over another would allow us to examine whether one source of information is privileged over another. Thus, for each duration condition we conducted three a-priori comparisons: Foreground vs. Inconsistent Control, Background vs. Inconsistent Control, and Foreground vs. Background responses. For all planned comparisons, alpha was adjusted such that the family-wise error did not exceed .05 . The specific alpha used is reported below prior to the report of the tests. ${ }^{2}$

As a manipulation check, we performed the analyses on the Normal scene condition $(\alpha=.004)$. As might be expected, we found that both the Foreground and Background target responses were significantly different from the Inconsistent condition, for both the $50 \mathrm{~ms}$ (Foreground: $t(35)=7.66, p<.001, d=1.81 ;$ Background: $t(35)=7.76, p<.001, d=1.72)$ and $100 \mathrm{~ms}$ (Foreground: $t(35)=20.92, p<.001, d=3.76$; Background: $t(35)=13.44, p<.001, d=3.31$ ). This pattern showed that participants were engaged and able to complete the task.

Accordingly, we found no difference in responses between Foreground and Background for either the 50ms, $t(35)=.40, p=.692, d=.07$, or $100 \mathrm{~ms}$ conditions, $t(35)=1.21, p=.233, d=.25$. This is expected as regardless of whether the foreground or background is initially used for the

\footnotetext{
${ }^{2}$ For each contrast, we report Cohen's d (Cohen, 1988), based on the within-subject formula.
} 
interpretation, the responses would be the same. Thus, because the Normal condition was not of theoretical interest, it will not be discussed or reported further. Means are included in all charts for posterity. 


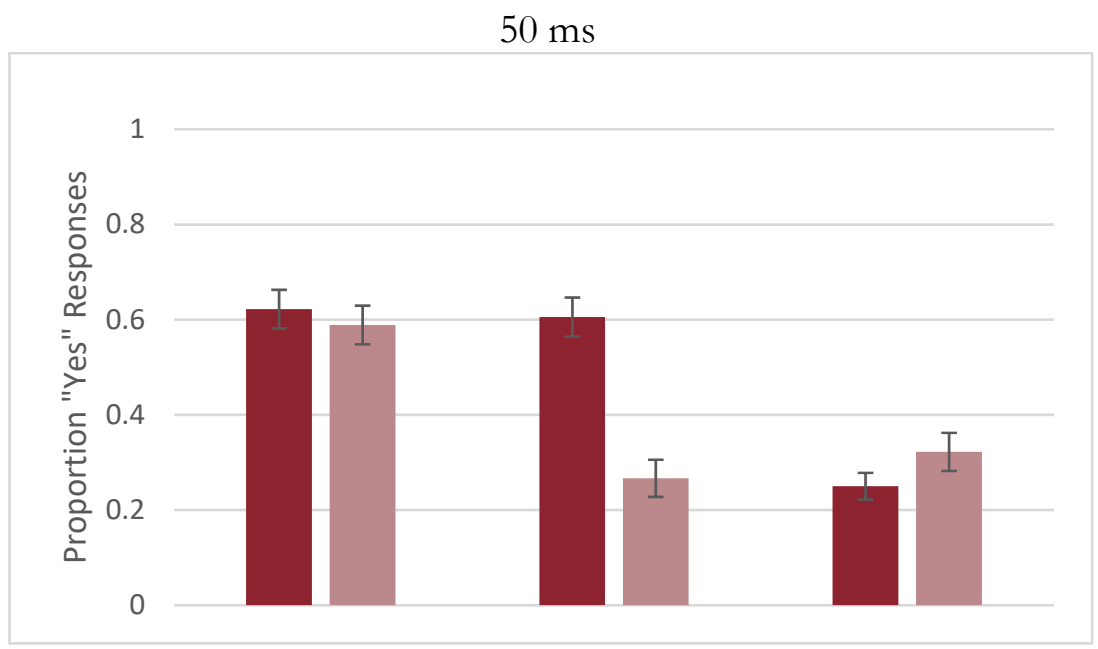

$250 \mathrm{~ms}$
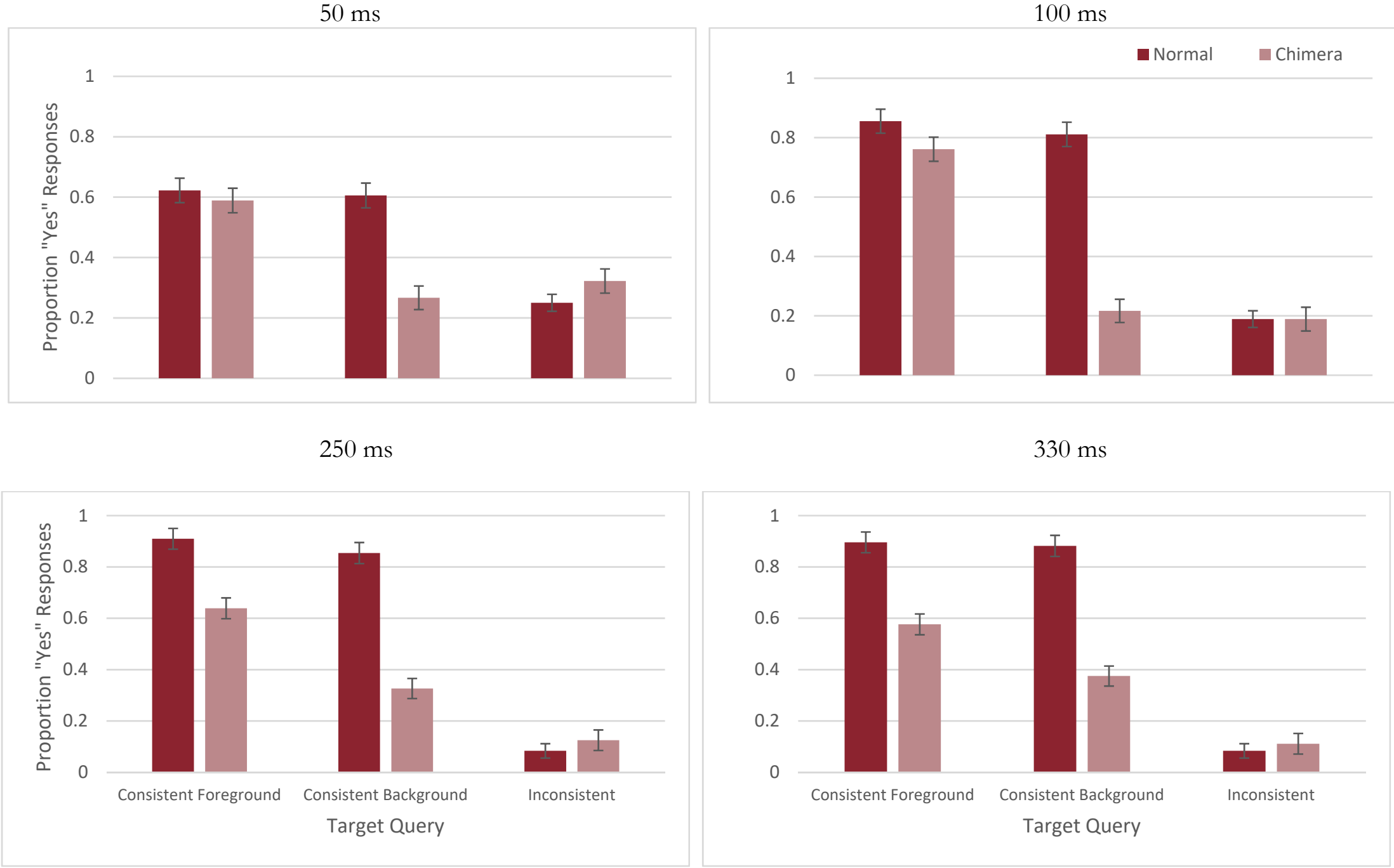

$330 \mathrm{~ms}$

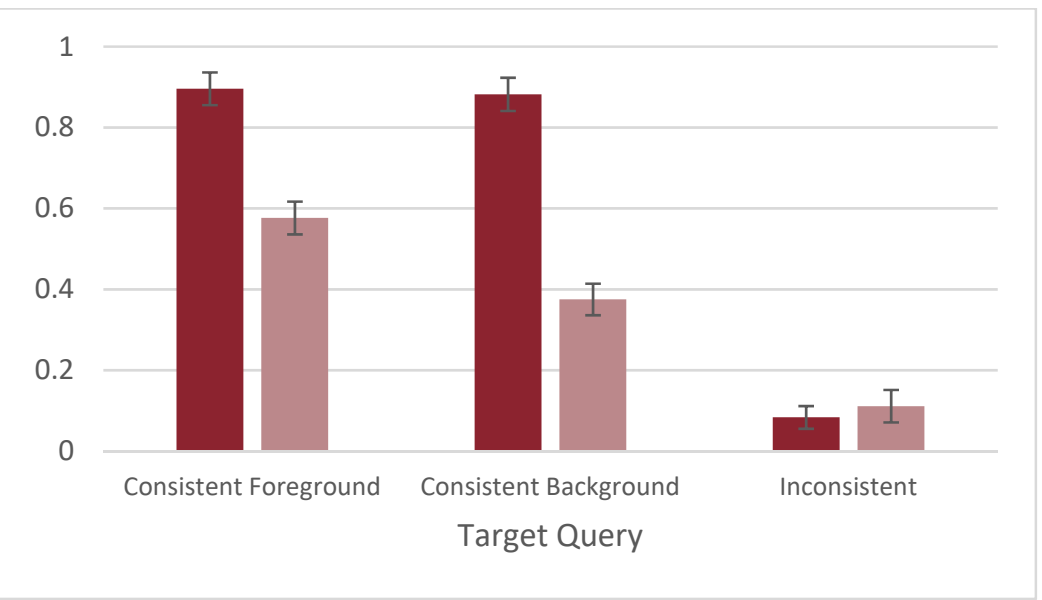

Figure 3. The mean proportion "yes" responses for Normal and Chimera scenes for each of the Target query conditions for Experiment 1 (target was an object that was not present in the scene; see text for details). Error bars represent Standard Error of the Mean. 
The mean proportion of "yes" responses across the Scene Type and Target conditions are shown in top row of Figure 2 for Experiment 1 a. As mentioned above, planned comparisons were done on the Chimera scenes as these images contained the theoretically interesting contrast between scene categories within a single image. The paired sample t-tests showed that participants interpreted the scene differently across duration conditions (6 comparisons, $\alpha=.00833)$. When we compared the Foreground and Background target conditions to the Inconsistent Control condition, we found at $50 \mathrm{~ms}$, participants were responding "yes" to the Foreground more than the Control, $t(35)=4.40$, $p<.001, d=1.10$, but interestingly did not show a significant difference in responses between the Background and Control, $t(35)=-1.28, \mathrm{p}=.21, d=-.23$. The pattern persisted in the $100 \mathrm{~ms}$ condition, such that Foreground condition had significantly more "yes" responses than the Control condition, $t(35)=15.26, p<.001, d=3.06$, but the Background condition did not, $t(35)=.71, p=.48, d=.15$. To assess the bias directly, we compared Foreground and Background target conditions directly and found a significantly greater proportion of responses for the Foreground than the Background for both $50 \mathrm{~ms}, t(35)=6.15, p<.001, d=1.35$, and $100 \mathrm{~ms}, t(35)=12.35, p<.001, d=3.03$.

The mean proportion of "yes" responses across the Scene Type and Target conditions are shown in the bottom row of Figure 2 for Experiment 1b. As above, we conducted pre-planned comparisons across target conditions at each duration condition (250 and 330ms) for the Chimera scenes (6 comparisons, $\alpha=.00833$ ). Participants were able to distinguish the Foreground from the inconsistent Control, for both $250 \mathrm{~ms}, t(35)=8.49, p<.001, d=2.33$, and $330 \mathrm{~ms}, t(35)=10.41$, $p<.001, d=2.63$. Additionally, in contrast to the results of Experiment 1a, participants were also able to distinguish the Background from the Inconsistent Control, for both $250 \mathrm{~ms}, t(35)=3.70$, $p=.001, d=1.00$, and $330 \mathrm{~ms}, t(35)=5.01, p<.001, d=1.36$. When the Foreground and Background conditions were contrasted directly, the results showed that participants remained significantly 
biased towards the foreground for both $250 \mathrm{~ms}, t(35)=5.07, p<.001, d=1.21$, and $330 \mathrm{~ms}, t(35)=4.23$, $p<.001, d=.87$.

\section{Experiment 2}

When we examined fixation placement, we found the central position often overlapped with the foreground scene region (78\%). Thus, the information at the fixation could explain the results. In Experiment 2, we manipulated where the participants were looking where the scene was presented by manipulating the position of the initial fixation prior to the image presentation.

\section{Methods}

Participants. Two groups of 48 participants took part in Experiments $2 \mathrm{a}$ and $2 \mathrm{~b}$ for course credit or were paid $\$ 10 /$ hour. All participants had normal or corrected-to-normal vision and none participated in the previous experiment. Sample size was determined using the effect size contrasting the Foreground vs. Background from Experiment 1, which was a large effect size $(d=.87-3.3)$ across duration conditions. Because we were interested in determining the effects of fixation position on interpretation, for our sample size computation ( $G^{*}$ power, Faul, Erdfelder, Lang, \& Buchner, 2007) we used a medium effect size of .6, $\alpha=.004$ (Bonferroni corrected for current study), and power at .8. The estimated sample size was 33 participants, but to counterbalance we recruited 48 , which yielded a power estimate of .86 .

Design. A 2-level factor manipulating fixation placement was added to create a 2 (initial fixation position: background, foreground) x 2 (presentation duration) x 2 (scene type: Normal, Chimera) x 3 (target condition: Foreground, Background, Inconsistent Control) within-subjects design $^{3}$. The presentation duration was $50 \mathrm{~ms}$ and $100 \mathrm{~ms}$ for Experiment $2 \mathrm{a}$ and was $250 \mathrm{~ms}$ and $330 \mathrm{~ms}$ for Experiment $2 \mathrm{~b}$.

\footnotetext{
${ }^{3}$ Due to the limited number of chimera scenes, we had 2 items/condition. This rather low number of data points per condition was not ideal, but was offset by increasing the number of participants per condition, using a completely within-subjects design, and adopting conservative analyses (Murphy, Myors, Wolach, Myors, \& Wolach, 2014).
} 
Procedure. The procedure was identical to Experiment 1, with the exception of the placement of the fixation cue. The fixation cue was placed either in the foreground or general background region of the scene. The position was a pre-determined location for each scene individually.

\section{Results}

Analysis. Again, the mean proportion of times participants indicated "Yes" to the match between the target word and the scene was calculated and used as the dependent variable in all analyses. For all planned comparisons, alpha was adjusted such that the family-wise error did not exceed $\alpha=.05$. The specific alpha used is reported below prior to the report of the tests. As each group of participants were collected at different times, each of the Experiments $2 \mathrm{a}$ and $2 \mathrm{~b}$ analyses were conducted separately; however exploratory analysis across both experiments can be found in the Supplementary Material. The means of the results are found in Figure 3. 

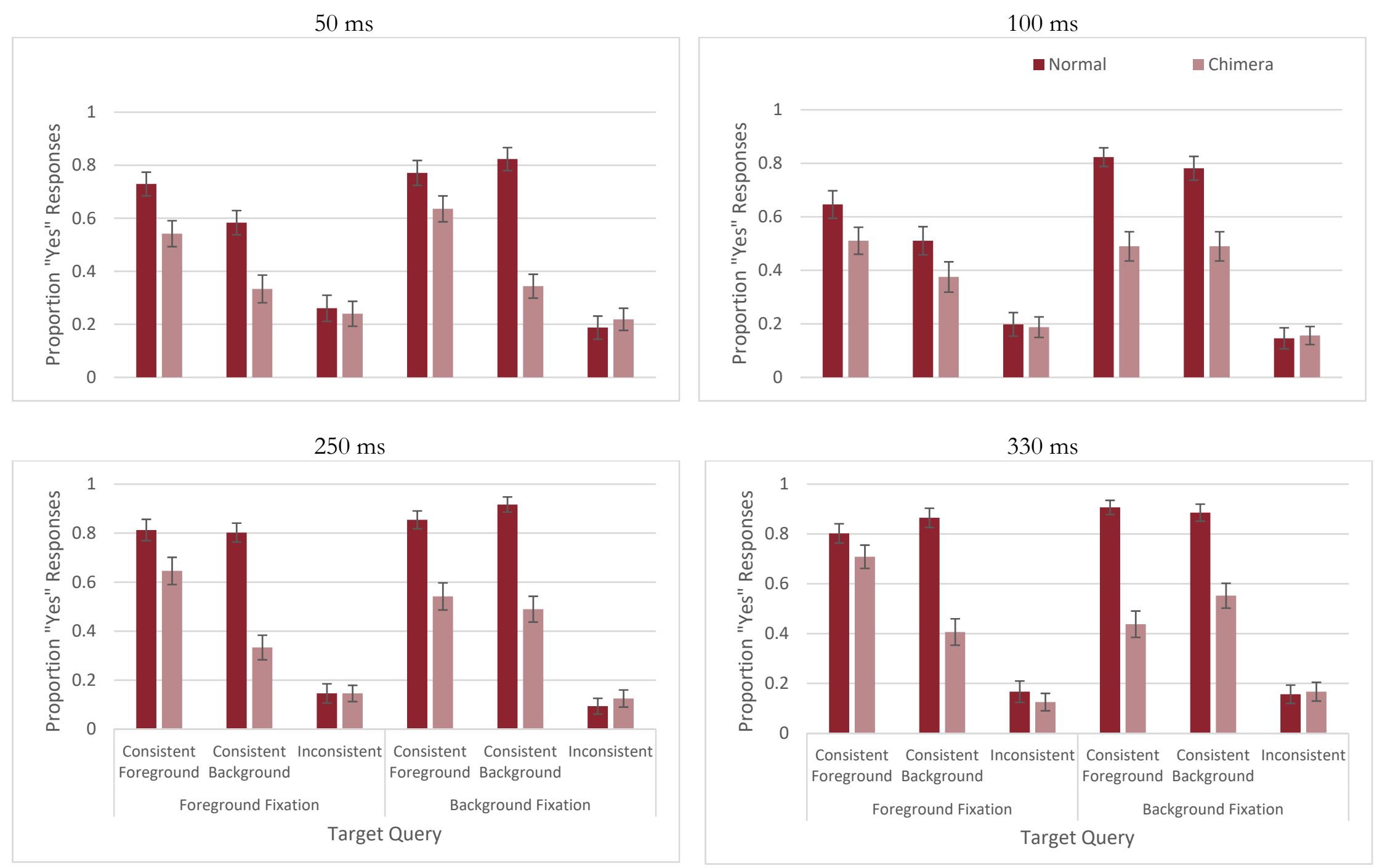

Figure 3. The mean proportion "yes" responses for Normal and Chimera scenes for each of the Target query (object not present in the scene; see text for details) and Fixation conditions for Experiment 2. Error bars represent Standard Error of the Mean. 
The mean proportion of "yes" responses across the Scene Type, Target and Fixation conditions are shown in the top row of Figure 3 for Experiment 2a. To examine the effect on the response bias, we conducted pre-planned comparisons of the Foreground and Background target conditions against the Inconsistent Control condition for each duration condition at each fixation position (12 comparisons, $\alpha=.004167)$. As might be expected, when the fixation was positioned on the foreground, the pattern of responses did not change from the first experiment. For both $50 \mathrm{~ms}$ and $100 \mathrm{~ms}$ duration conditions, participants responded "yes" to the Foreground more than the Control, $(t(47)=4.69, p<.001, d=.91$ and $t(47)=5.51, p<.001, d=1.05$, respectively $)$ Interestingly, they did not show a significant difference in responses between the Background and Control for 50ms durations, $(t(47)=1.46, p=.15, d=.27)$, but did show a significant difference with a medium size effect in the $100 \mathrm{~ms}$ condition $(t(47)=3.10, p=.003, d=.57)$. When we compare the responses from Foreground to the Background directly, we found that participants had a significant bias to the foreground targets for the $50 \mathrm{~ms}$ duration, but there was no significant difference for the $100 \mathrm{~ms}$ duration $(t(47)=3.00, p=.004, d=.60$ and $t(47)=1.91, p=.063, d=.36$, for $50 \mathrm{~ms}$ and $100 \mathrm{~ms}$ respectively). Thus, compared to the previous experiment, the effect was diminished but persisted.

Surprisingly, when the fixation was in the background, we found that participants continued to show the Foreground Bias. We found participants responded "yes" to the Foreground target more than the Inconsistent Control, $(t(47)=6.93, p<.001, d=1.32$ and $t(47)=4.85, p<.001, d=1.09$, for $50 \mathrm{~ms}$ and $100 \mathrm{~ms}$ respectively), but did not show a significant difference in responses between the Background and inconsistent Control for 50ms durations, $(t(47)=1.95, p=.057, d=.42)$. However, there was a difference in the $100 \mathrm{~ms}$ condition $(t(47)=5.38, p<.001, d=1.09)$.

When we compare the responses from Foreground to the Background directly, we found that participants showed a significant bias for the foreground targets at 50ms, $t(47)=4.64, p<.001$, $d=.90$, but not at $100 \mathrm{~ms}$ where the means were almost identical, $t(47)<1$. Therefore, these results 
showed that a strong bias to the foreground remained early on, even when the fixation cue location varied. While not surprisingly there was a Foreground Bias when the fixation location was in the foreground, the bias never reversed to the background of a scene when fixating the background directly. Instead, the Foreground Bias was present with short exposures $(50 \mathrm{~ms})$ and then, with increased exposure, responses were equally likely between the foreground and background information. To further assess the effects of fixation location, and to see if the effect would eventually reverse, we conducted another experiment with longer exposure durations: 250 and $330 \mathrm{~ms}$.

The mean proportion of "yes” responses across the Scene Type, Target and Fixation conditions are shown in the bottom row of Figure 3 for Experiment $2 \mathrm{~b}$. To assess whether the bias to the foreground of scenes continued with longer presentation times, we conducted the three preplanned comparisons contrasting the Foreground and Background target conditions as before for each duration condition at each fixation location (12 comparisons, $\alpha=.004167)$. When the fixation was positioned on the foreground, we found that participants responded "yes" to the Foreground more than the Control, $(t(47)=7.51, p<.001, d=1.63$ and $t(47)=10.01, p<.001, d=2.06$, for $250 \mathrm{~ms}$ and $330 \mathrm{~ms}$ respectively), and to the Background more than the Control, $(t(47)=3.00, p=.004, \mathrm{~d}=.65$ and $t(47)=4.23, p<.001, d=.92$, for $250 \mathrm{~ms}$ and $330 \mathrm{~ms}$, respectively). Additionally, when we compare the responses from Foreground to the Background directly, we found that participants still had a significant bias to the foreground targets for both duration presentations $(t(47)=4.15, p<.001$, $d=.85$ and $t(47)=4.46, p<.001, d=.85$, for $250 \mathrm{~ms}$ and $330 \mathrm{~ms}$, respectively).

The pattern was somewhat different when the fixation was in the background. We found that participants responded "yes" to the Foreground more than the Control, $(t(47)=6.37, p<.001$, $d=1.33$ and $t(47)=3.97, p<.001, d=.87$, for $250 \mathrm{~ms}$ and $330 \mathrm{~ms}$ duration conditions respectively) and to the Background target more than the Control $(t(47)=6.38, p<.001, d=1.20$ and $t(47)=5.90$, 
$p<.001, d=1.27$, for $250 \mathrm{~ms}$ and $330 \mathrm{~ms}$ duration conditions respectively). When compared directly, we found that in neither duration condition did participants showed a significant bias for the foreground targets $(t(47)=.636, p=.528, d=.14$ and $t(47)=-1.50, p=.14, d=-.32$, for $250 \mathrm{~ms}$ and $330 \mathrm{~ms}$ duration conditions respectively). Instead, it seems that participants were responding to the foreground and background target in equal measure. Interestingly, although the Background fixation did eliminate the Foreground Bias, it was never reversed such that background information was favoured significantly over foreground information. This suggests that processing of the foreground is mitigated by fixation position, but also that the even when the background information is fixated, the foreground continues to exert a strong influence on responses.

\section{Experiment 3}

Although the Foreground bias seems to be quite robust, the previous experiments required participants to respond to queries about the presence of objects, which may have inadvertently encouraged participants to strategically focus on the foreground. To examine whether Foreground Bias resulted from the task, we conducted a third experiment examining participants' responses to the scene category name.

\section{Methods}

Participants. Two groups of thirty-six participants each provided informed consent and took part in Experiment 3 for course credit or were given $\$ 10 /$ hour for their time. All participants had normal or corrected-to-normal vision and none participated in previous experiments. The sample size was based on Experiment 1, as the design and effects of interest were identical.

Design. The design was similar to Experiment 1, with a 2 (presentation duration) x 2 (scene type: Normal, Chimera) x 3 (target condition: consistent-background, consistent-foreground, 
inconsistent) within-subjects design. As above, Experiment 3a presented scenes for $50 \mathrm{~ms}$ or 100ms; Experiment $3 \mathrm{~b}$ presented scenes for $250 \mathrm{~ms}$ or $330 \mathrm{~ms}$.

Procedure. The procedure was identical to Experiments 1 and 2, with the exception of the query: Instead of a target object word appearing after the scene, a scene category name was presented. The scene query belonged to one of three conditions: (i) background scene category, (ii) foreground scene category, or (iii) Inconsistent with either the background or the foreground scene category. The participants' task was to indicate whether the scene category word matched the image using a Yes/No button-press.

\section{Results}

Analysis. The mean proportion of times a participant indicated "Yes" the scene category matched the scene was calculated and used as the dependent variable for all analyses. As each group of participants was collected at different times, each of the Experiment $3 \mathrm{a}$ and $3 \mathrm{~b}$ analyses were conducted separately; however exploratory analysis across both experiments can be found in the Supplementary Material. The means of the results are found in Figure 4. 
$50 \mathrm{~ms}$

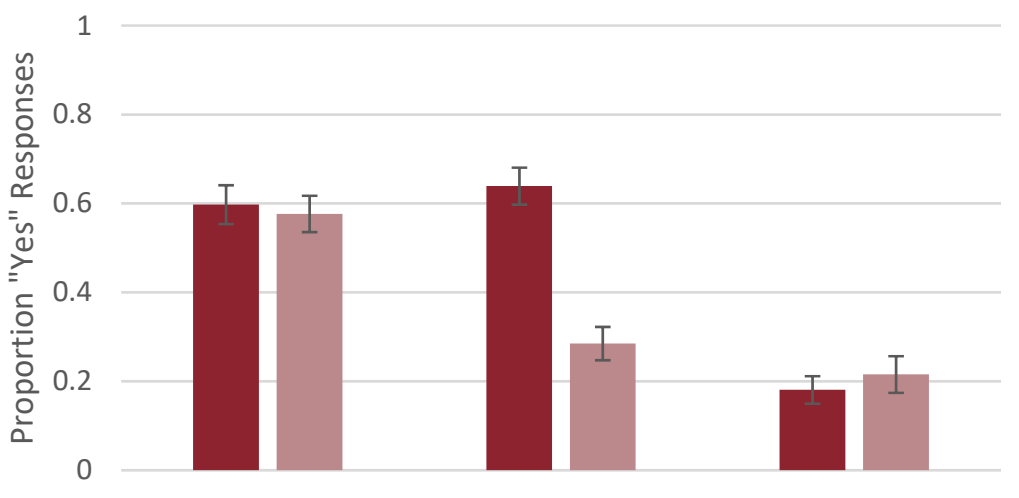

$250 \mathrm{~ms}$

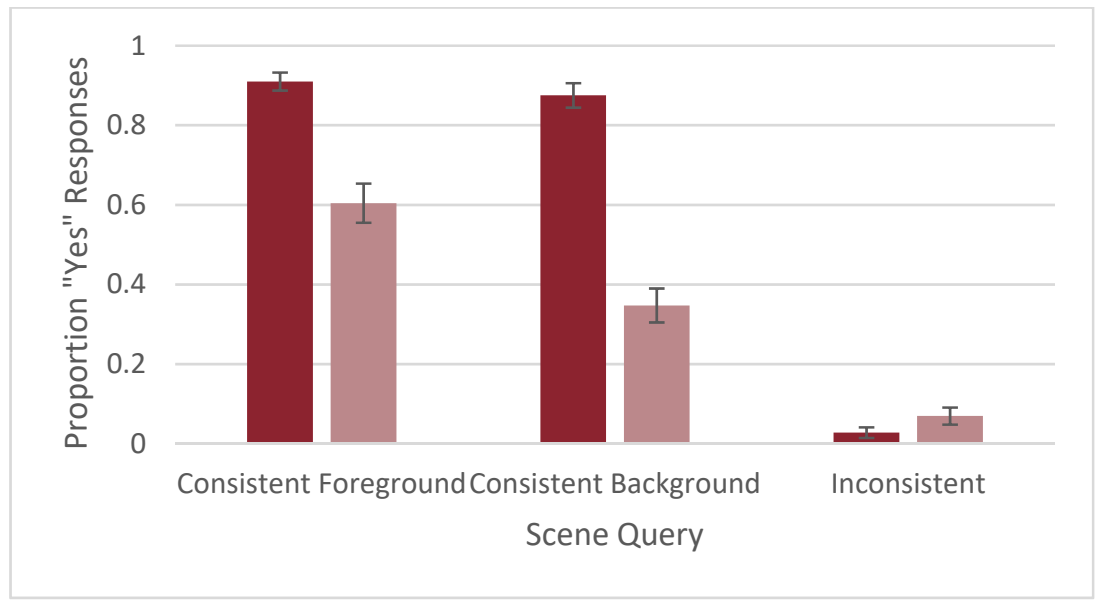

$100 \mathrm{~ms}$

- Norma

Chimera

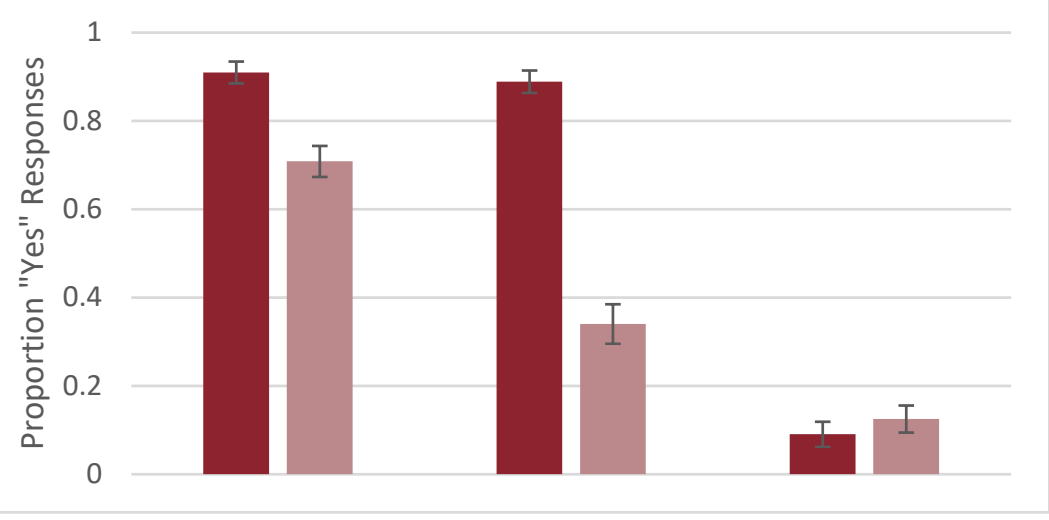

$330 \mathrm{~ms}$

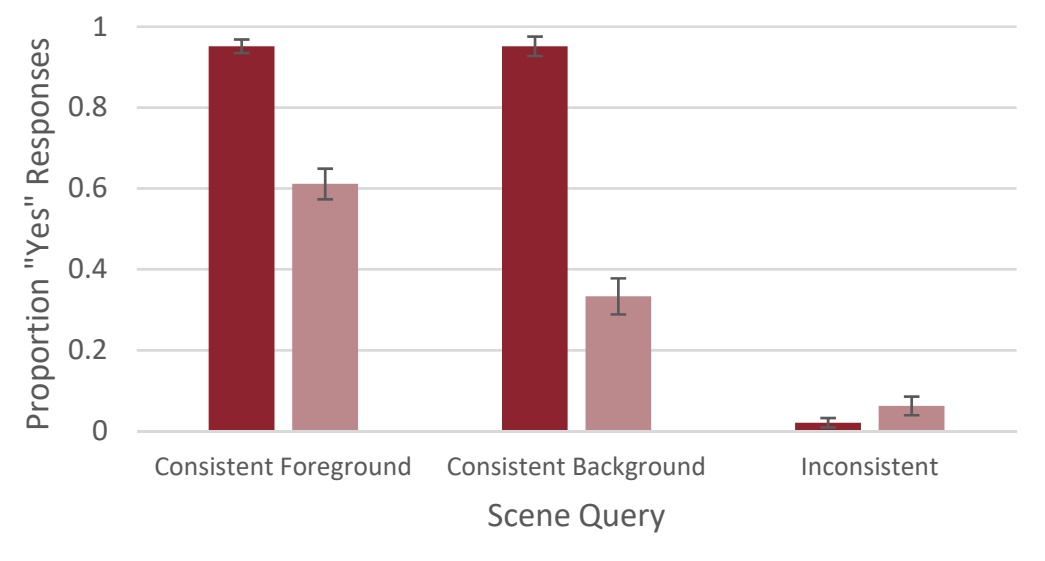

Figure 4. The mean proportion "yes" responses for Normal and Chimera scenes for each of the Scene query conditions for Experiment 3. Error bars represent Standard Error of the Mean. 
The mean proportion of "yes" responses across the Scene Type and Scene conditions are shown in the top row of Figure 4 for Experiment 3a. Planned comparisons using paired sample ttests showed that when responding to scene category names, the patterns of response were similar to Experiment 1 (6 comparisons, $\alpha=.00833$ ). When we compared the Foreground and Background scene conditions to the Inconsistent scene query condition, we found at $50 \mathrm{~ms}$, participants were responding "yes" to the Foreground more than the Control, $t(35)=5.93, p<.001, d=1.47$, but interestingly did not show a significant difference in responses between the Background and Control, $t(35)=1.30, p=.20, d=.29$. Interestingly, the pattern for $100 \mathrm{~ms}$ changed such that both Foreground and Background conditions had significantly more "yes" responses than the Control condition, $t(35)=11.07, p<.001, d=2.94$, and $t(35)=5.073, p<.001, d=.95$, respectively. To assess the Foreground Bias, we compared responses to Foreground and Background scene query conditions and found a significantly greater proportion of responses for the Foreground than the Background for both $50 \mathrm{~ms}, t(35)=4.78, p<.001, d=1.24$, and $100 \mathrm{~ms}, t(35)=5.67, p<.001, d=1.53$. Thus, even when the judgement was about the scene, the Foreground Bias persisted. To determine whether this bias would be affected by longer exposure, we showed scenes for 250 and $330 \mathrm{~ms}$.

The mean proportion of "yes" responses across the Scene Type and Scene conditions are shown in the bottom row of Figure 4 for Experiment $3 \mathrm{~b}$. When shown for $250 \mathrm{~ms}$ and $330 \mathrm{~ms}$, planned comparisons using paired sample t-tests (6 comparisons, $\alpha=.00833$ ) showed a similar pattern of results to the $100 \mathrm{~ms}$ duration condition. When we compared the Foreground and Background scene query conditions to the Inconsistent scene query condition for $250 \mathrm{~ms}$, we found significantly more "yes" responses, $t(35)=10.70, p<.001, d=2.35$, and $t(35)=5.72, p<.001, d=1.37$, for the Foreground and Background respectively. The same pattern was found for $330 \mathrm{~ms}, t(35)=14.82$, $p<.001, d=2.92$, and $t(35)=6.52, p<.001, d=1.27$, for the Foreground and Background respectively. 
To assess the Foreground Bias, we compared responses to Foreground and Background scene query conditions and found a significantly greater proportion of responses for the Foreground than the Background for both $250 \mathrm{~ms}, t(35)=3.50, p<.001, d=1.24$, and $330 \mathrm{~ms}, t(35)=4.66, p<.001, d=.93$. Thus, even with longer presentation durations and when judging the scene category, the Foreground Bias persisted.

\section{General Discussion}

Across three experiments, we found that when given conflicting information across depth, participants were initially more influenced by the scene foreground than background. Experiment 1 showed that the Foreground Bias was present for $50 \mathrm{~ms}$ and $100 \mathrm{~ms}$ exposure durations, but dissipated with longer durations. In Experiment 2, when the fixation position overlapped with background of the scenes, the Foreground Bias remained with brief exposure durations (50ms), and was weaken $(100 \mathrm{~ms})$ or absent with longer presentation durations (250ms and $330 \mathrm{~ms})$. It is interesting to note that we never saw participants show a preference for the background, even when it was directly fixated. We discuss possible reasons below. In Experiment 3, we modified the task to encourage focus on the whole scene and found the task only slightly modified the pattern of results. The Foreground Bias remained, but the background was now correctly interpreted with slightly longer exposures $(100 \mathrm{~ms})$. Interestingly, across experiments we never saw a reverse effect favoring background information, suggesting the Foreground Bias effect was robust.

\section{Central Bias vs. Foreground Bias}

The central bias is a well-established phenomenon in scene perception (Bindemann, 2010; Foulsham \& Kingstone, 2013; Rothkegel, Trukenbrod, Schütt, Wichmann, \& Engbert, 2017; Tatler, 2007). The bias reflects the tendency to direct fixations to the centre of an image. Researchers posit that there are two potential sources for this central bias (Bindemann, 2010). First, the central position allows for the maximal perception of information across the whole scene. Thus, for a scene 
image displayed on a monitor, the center represents an optimal viewing position, such as has been found with words and objects (McConkie, Kerr, Reddix, \& Zola, 1988; Pajak \& Nuthmann, 2013; Vitu, O’Regan, \& Mittau, 1990).

In Experiment 2, we examined the central bias based on this optimal viewing position notion. If the central bias was responsible for this effect because the information at the centre overlapped with the foreground, then we would expect the foreground bias to disappear once attention is directed elsewhere (and be dominated by the information at the current fixation, whether in the foreground or background). However, if the central bias is an effect orthogonal to the foreground bias such that both have an effect on scene gist processing, then we would expect overall scene gist perception to decrease once the fixations are not centred, but processing differences between foreground and background to persist. Our results are consistent with the latter. Thus, although we do not dispute that the central bias may also play a role in the perception of scene gist, we believe the foreground bias is an important separate effect in initial scene processing.

However, the central bias has also been described as an inherent property of the scene itself, where information at the centre is driving the effect (Bindemann, 2010; Tatler, 2007). For instance, in the case of photographs, semantically important information is often centred in the image (Bindemann, 2010; Rensink, O’Regan, \& Clark, 1997). To test this interpretation more directly, it would require a change the contents of each scene such that none of the foreground information were located in the centre (e.g., by changing viewpoints, Castelhano \& Pollatsek, 2010; Castelhano, Pollatsek, \& Rayner, 2009). Although we did not have this manipulation, we did a further examination of Experiment 1a data with a post-hoc analysis of the foreground bias with scenes in which the foreground was not in the centre. We found that the pattern of the means was consistent with the results across all experiments, although fewer data points meant the pattern of results was more uneven (see Supplementary Materials for report of full post-hoc analyses). For instance, we 
found for $100 \mathrm{~ms}$ a significant difference between the responses to the foreground target and control, between foreground targets and background targets, but not between the background targets and the control. Although this post-hoc analysis suggests that the foreground bias may be orthogonal to the central bias, further investigation into how these two effects are related is necessary.

\section{Attention Allocation and Scene Gist Perception}

Inherent in the arguments of the central bias is how attention changes over time. For instance, when considering Experiment 2, one interpretation of the pattern across duration conditions is that processing is more influenced by information at fixation over time. Many previous studies have shown that the focus of attention is initially diffuse (Greene \& Oliva, 2009b; Henderson, 2003; Aude Oliva \& Torralba, 2006) and then becomes more focused over time (Castelhano \& Henderson, 2003, 2007; Henderson, Williams, Castelhano, \& Falk, 2003; Schyns \& Oliva, 1994). In the current study, this meant that at short durations there was little effect of the information at fixation, and the effects of information at fixation became discernible only with longer durations. Alternatively, one could interpret the same pattern as the ever increasing influence of background information as more time for processing is allowed. For instance, the pattern shows a decrease in responses for the Foreground target as more time for processing is allowed, presumably allowing incorporation of the contradiction between the Foreground and the Background to take place. The question of how attention or information prioritization changes over time would require further research to delineate how this bias and attentional processes interact.

\section{Flexibility of the Scene Representation}

Past research has noted the flexibility of scene representations (Castelhano et al., 2018; deGraef et al., 1990; Malcolm, Nuthmann, \& Schyns, 2014; Oliva \& Schyns, 1997; Schyns \& Oliva, 1994). For instance, Malcom et al. (2014) showed that depending on the level of categorization, 
different types of visual information were used. They found that when the categorization was at a subordinate-level, more detailed information about specific objects were used, in contrast to the basic-level categorization. The flexibility has also been reflected in what type of information is processed over time. de Graef et al., (1990) found that the effect of scene context on object recognition was not observable until later stages of processing (after a number of fixations). In the current study, the timing of the shift from foreground to background changed with task, between when the task was more object-based vs. scene-based. This type of flexibility seems to be akin to that also demonstrated by Oliva and Schyns (1997). While Schyns and Oliva (1994) demonstrated that scenes processing proceeds in a course-to-fine manner, Oliva and Schyns (1997) demonstrated that brief exposures of either low-pass or high-pass led to understanding. Thee current results also reflect the flexibility of the visual representation, and as changes to the timing and extent of the Foreground Bias changed with presentation duration and task. Thus, the Foreground Bias may be reflection of the same type of flexibility as past studies.

\section{Spatial Information Processing in Scenes}

The current study is a first step in examining how the z-axis of scenes impacts initial processing. Researchers have begun to examine information across different depths in scenes (Bonner \& Epstein, 2017, 2018; Josephs \& Konkle, 2019). Taken together with the current results, it seems the immediately surrounding scene space within which we can plan and execute actions (actionable space) may be prioritized early on and processed differently compared to more distant scene regions.

In the depth perception literature, many researchers categorize the information available at different depths as categories of space linked to specific types of processing (Armbrüster, Wolter, Kuhlen, Spijkers, \& Fimm, 2008; Costantini et al., 2011; Cutting \& Vishton, 1995; Previc, 1998). For instance, space can be divided into peripersonal, actionable and vista space (Costantini et al., 
2011; Cutting \& Vishton, 1995). Across these categories, researchers have found different depth cues are extracted, such as motion perspective (i.e., motion parallax), height (i.e., in the field), and relative density (i.e., texture gradient), respectively. Thus, there are functionally different types of information available across depth. Furthermore, this categorization of spatial proximity introduces an interesting framework from which to consider the nexus of processing scene context, objects, and actions (e.g., Bonner \& Epstein, 2017; M.S. Castelhano \& Pereira, 2018; Monica S. Castelhano \& Witherspoon, 2016; Greene, Baldassano, Esteva, Beck, \& Fei-Fei, 2016; Josephs \& Konkle, 2019).

\section{Influence of the Foreground}

The current study suggests that the Foreground Bias may extend to later processing. Although the response bias for the foreground dissipated with increased exposure, it was never reversed, even when the background was directly fixated or the task was changed to focus on the scene as a whole. Thus, it seems that the influence of foreground continued. This is consistent with the few studies examining foreground and background processing in other tasks. For instance, Mazza, Turatto and Umiltá (2005) found in a change detection task that changes were detected more quickly in the scene foreground than background. More recently, we found that during visual search, target objects were found faster when located in the foreground than the background (even when object size was controlled; Man \& Castelhano, 2018). Together, these studies suggest that prioritizing the foreground may continue to exert influence in later processing, but further research is needed to delineate its extent.

\section{Conclusions}

The current results have implications for models of scene representations and the representations of an observer's immediate surrounding space. The present study suggests that consideration of different categories of space across depth offers a framework within which different types of scene processing can be understood. With an increased accessibility to techniques 
such as Virtual Reality (VR) environments, we believe this theoretical approach could offer new insights into exploring the visual processing in the real-world and processing differences across the z-axis of scenes. 


\section{Acknowledgements}

The authors thank Ellen O’Donoghue, Louisa Man, Mollie Roy, Morgan Vallati, Elysée Kukwabantu, and Shane Kennedy for their help. This work was supported by funding from the Natural Sciences and Engineering Research Council of Canada, Canadian Foundation for Innovation, and Ontario Ministry of Research and Innovation to MSC. 


\section{References}

Armbrüster, C., Wolter, M., Kuhlen, T., Spijkers, W., \& Fimm, B. (2008). Depth Perception in Virtual Reality: Distance Estimations in Peri- and Extrapersonal Space. CyberPsychology \& Behavior, 11(1), 9-15. https://doi.org/10.1089/cpb.2007.9935

Bindemann, M. (2010). Scene and screen center bias early eye movements in scene viewing. Vision Research, 50(23), 2577-2587. https://doi.org/10.1016/J.VISRES.2010.08.016

Bonner, M. F., \& Epstein, R. A. (2017). Coding of navigational affordances in the human visual system. Proceedings of the National Academy of Sciences of the United States of America, 114(18), 47934798. https://doi.org/10.1073/pnas.1618228114

Bonner, M. F., \& Epstein, R. A. (2018). Computational mechanisms underlying cortical responses to the affordance properties of visual scenes. PLOS Computational Biology, 14(4), e1006111. https://doi.org/10.1371/journal.pcbi.1006111

Brainard, D. H. (1997). The Psychophysics Toolbox. Spatial Vision, 10(4), 433-436. https://doi.org/10.1163/156856897X00357

Castelhano, M.S., \& Pereira, E. J. (2018). The influence of scene context on parafoveal processing of objects. Quarterly Journal of Experimental Psychology, 71(1 Special Issue). https://doi.org/10.1080/17470218.2017.1310263

Castelhano, M. S., Fernandes, S., \& Theriault, J. (2018). Examining the hierarchical nature of scene representations in memory. Journal of Experimental Psychology: Learning, Memory, and Cognition, Manuscript in press. https://doi.org/10.1037/xlm0000660

Castelhano, M. S., \& Henderson, J. M. (2003). Flashing scenes and moving windows: An effect of initial scene gist on eye movements. Journal of Vision, 3(9), 67a. https://doi.org/10.1167/3.9.67

Castelhano, M. S., \& Henderson, J. M. (2007). Initial scene representations facilitate eye movement guidance in visual search. Journal of Experimental Psychology: Human Perception and Performance, 
33(4), 753-763. https://doi.org/10.1037/0096-1523.33.4.753

Castelhano, M. S., \& Henderson, J. M. (2008). The influence of color on the perception of scene gist. Journal of Experimental Psychology: Human Perception and Performance, 34(3), 660-675. https://doi.org/10.1037/0096-1523.34.3.660

Castelhano, M. S., \& Witherspoon, R. L. (2016). How You Use It Matters: Object Function Guides Attention During Visual Search in Scenes. Psychological Science, 27(5), 606-621. https://doi.org/10.1177/0956797616629130

Castelhano, M. S, \& Pollatsek, A. (2010). Extrapolating spatial layout in scene representations. Memory Cognition, 38(8), 1018-1025. Retrieved from http://www.ncbi.nlm.nih.gov/pubmed/21156866

Castelhano, M. S, Pollatsek, A., \& Rayner, K. (2009). Integration of multiple views of scenes. Attention Perception Psychophysics, 71(3), 490-502. https://doi.org/10.3758/APP

Cohen, J. (1988). Statistical power analysis for the behavioral sciences (2nd ed.). Hillsdale N.J.: L. Erlbaum Associates.

Costantini, M., Ambrosini, E., Scorolli, C., \& Borghi, A. M. (2011). When objects are close to me: Affordances in the peripersonal space. Psychonomic Bulletin \& Review, 18(2), 302-308. https://doi.org/10.3758/s13423-011-0054-4

Cutting, J. E., \& Vishton, P. M. (1995). Perceiving layout and knowing distances: The integration, relative potency, and contextual use of different information about depth. In W. Epstein \& S. J. Rogers (Eds.), Handbook of perception and cognition (2nd ed., pp. 69-117). San Diego: Academic Press. https://doi.org/10.1016/B978-012240530-3/50005-5

Davenport, J. L., \& Potter, M. C. (2004). Scene Consistency in Object and Background Perception. Psychological Science, 15(8), 559-564. https://doi.org/10.1111/j.0956-7976.2004.00719.x

Evans, K., Horowitz, T., \& Wolfe, J. (2011). When categories collide: Accumulation of information 
about multiple categories in rapid scene perception. Psychological Science, 22(6), 739-746.

https://doi.org/https://doi.org/10.1177/0956797611407930

Faul, F., Erdfelder, E., Lang, A.-G., \& Buchner, A. (2007). G*Power 3: A flexible statistical power analysis program for the social, behavioral, and biomedical sciences. Behavior Research Methods, 39(2), 175-191. https://doi.org/10.3758/BF03193146

Foulsham, T., \& Kingstone, A. (2013). Optimal and Preferred Eye Landing Positions in Objects and Scenes. Quarterly Journal of Experimental Psychology, 66(9), 1707-1728.

https://doi.org/10.1080/17470218.2012.762798

Graef, P. De, Christiaens, D., d’Ydewalle, G. G., De Graef, P., Christiaens, D., \& d'Ydewalle, G. G. (1990). Perceptual effects of scene context on object identification. Psychological Research, 52(4), 317-329. https://doi.org/10.1007/BF00868064

Greene, M. R., Baldassano, C., Esteva, A., Beck, D. M., \& Fei-Fei, L. (2016). Visual scenes are categorized by function. Journal of Experimental Psychology. General, 145(1), 82-94. https://doi.org/10.1037/xge0000129

Greene, M. R., \& Oliva, A. (2009a). Recognition of natural scenes from global properties: Seeing the forest without representing the trees. Cognitive Psychology, 58(2), 137-176. https://doi.org/10.1016/j.cogpsych.2008.06.001

Greene, M. R., \& Oliva, A. (2009b). The Briefest of Glances: The Time Course of Natural Scene Understanding. Psychological Science, 20(4), 464-472. https://doi.org/10.1111/j.14679280.2009.02316.x

Greene, M. R., \& Oliva, A. (2010). High-level aftereffects to global scene properties. Journal of Experimental Psychology: Human Perception and Performance, 36(6), 1430-1442. https://doi.org/10.1037/a0019058

Henderson, J. M. (2003). Human gaze control during real-world scene perception. Trends in Cognitive 
Sciences, 7(11), 498-504. https://doi.org/10.1016/j.tics.2003.09.006

Henderson, J. M., \& Hollingworth, A. (1999). High-level scene perception. Annual Review of Psychology, 50(1), 243-271. https://doi.org/10.1146/annurev.psych.50.1.243

Henderson, J. M., Williams, C. C., Castelhano, M. S., \& Falk, R. J. (2003). Eye movements and picture processing during recognition. Perception \& Psychophysics, 65(5), 725-734. https://doi.org/10.3758/BF03194809

Josephs, E. L., \& Konkle, T. (2019). Perceptual dissociations among views of objects, scenes, and reachable spaces. Journal of Experimental Psychology: Human Perception and Performance, 45(6), 715728. https://doi.org/10.1037/xhp0000626

Koehler, K., \& Eckstein, M. P. M. (2017). Beyond scene gist: Objects guide search more than scene background. Journal of Experimental Psychology:, 43(6), 1177-1193. https://doi.org/10.1037/xhp0000363

Malcolm, G. L., Nuthmann, A., \& Schyns, P. G. (2014). Beyond gist: strategic and incremental information accumulation for scene categorization. Psychological Science, 25(5), 1087-1097. https://doi.org/10.1177/0956797614522816

Man, L. L. Y., \& Castelhano, M. S. (2018). Across the planes: Differing impacts of foreground and background information on visual search in scenes. Journal of Vision, 18(10), 384. https://doi.org/10.1167/18.10.384

Mazza, V., Turatto, M., \& Umilta, C. (2005). Foreground?background segmentation and attention: A change blindness study. Psychological Research Psychologische Forschung, 69(3), 201-210. https://doi.org/10.1007/s00426-004-0174-9

McConkie, G. W., Kerr, P. W., Reddix, M. D., \& Zola, D. (1988). Eye movement control during reading: I. The location of initial eye fixations on words. Vision Research, 28(10), 1107-1118. https://doi.org/10.1016/0042-6989(88)90137-X 
Munneke, J., Brentari, V., \& Peelen, M. V. (2013). The influence of scene context on object recognition is independent of attentional focus. Frontiers in Psychology, 4, 552. https://doi.org/10.3389/fpsyg.2013.00552

Murphy, K. R., Myors, B., Wolach, A., Myors, B., \& Wolach, A. (2014). Statistical Power Analysis. Routledge. https://doi.org/10.4324/9781315773155

Nagata, S. (1993). How to reinforce perception of depth in single two-dimensional pictures. Pictorial Communication in Virtual and Real Environments, 527-545.

Oliva, A., \& Schyns, P. G. (1997). Coarse Blobs or Fine Edges? Evidence That Information Diagnosticity Changes the Perception of Complex Visual Stimuli. Cognitive Psychology, 34(1), 72 107.

Oliva, A., \& Torralba, A. (2001). Modeling the Shape of the Scene: A Holistic Representation of the Spatial Envelope. International Journal of Computer Vision, 42(3), 145-175.

Oliva, A. (2005). Gist of the scene. In L. Itti, G. Rees, \& J. K. Tsotsos (Eds.), Neurobiology of attention (pp. 251-256). Academic Press. https://doi.org/10.1016/B978-012375731-9/50045-8

Oliva, Aude, \& Torralba, A. (2006). Building the gist of a scene: the role of global image features in recognition. In Progress in Brain Research (Vol. 155, pp. 23-36). Elsevier. https://doi.org/10.1016/S0079-6123(06)55002-2

Pajak, M., \& Nuthmann, A. (2013). Object-based saccadic selection during scene perception: evidence from viewing position effects. Journal of Vision, 13(5). https://doi.org/10.1167/13.5.2

Pereira, E. J., \& Castelhano, M. S. (2014). Peripheral guidance in scenes: The interaction of scene context and object content. Journal of Experimental Psychology: Human Perception and Performance, 40(5), 2056-2072. https://doi.org/10.1037/a0037524

Pereira, E. J., \& Castelhano, M. S. (2019). Attentional capture is contingent on scene region: Using surface guidance framework to explore attentional mechanisms during search. Psychonomic 
Bulletin \& Review, in press. https://doi.org/10.3758/s13423-019-01610-z

Potter, M. C. (1975). Meaning in visual search. Science, 187, 965-966.

Potter, M. C. (1976). Short-term conceptual memory for pictures. Journal of Experimental Psychology: Human Learning and Memory, 2(5), 509-522. https://doi.org/1003124

Previc, F. H. (1998). The neuropsychology of 3-D space. Psychological Bulletin, 124(2), 123-164.

Rensink, R. A., O’Regan, J. K., \& Clark, J. J. (1997). To See or not to See: The Need for Attention to Perceive Changes in Scenes. Psychological Science, 8(5), 368-373. https://doi.org/10.1111/j.14679280.1997.tb00427.x

Rothkegel, L. O. M., Trukenbrod, H. A., Schütt, H. H., Wichmann, F. A., \& Engbert, R. (2017). Temporal evolution of the central fixation bias in scene viewing. Journal of Vision, 17(13), 3. https://doi.org/10.1167/17.13.3

Schyns, P. G., \& Oliva, A. (1994). Evidence for Time-and Spatial-Scale-Dependent Scene Recognition. Psychological Science, 5(4), 195-200.

Tatler, B. W. (2007). The central fixation bias in scene viewing: Selecting an optimal viewing position independently of motor biases and image feature distributions. Journal of Vision, 7(14), 4. https://doi.org/10.1167/7.14.4

Thorpe, S., Fize, D., \& Marlot, C. (1996). Speed of processing in the human visual system. Nature, 381(6582), 520-522. https://doi.org/10.1038/381520a0

Vitu, F., O’Regan, J. K., \& Mittau, M. (1990). Optimal landing position in reading isolated words and continuous text. Perception \& Psychophysics, 47(6), 583-600. https://doi.org/10.3758/BF03203111 